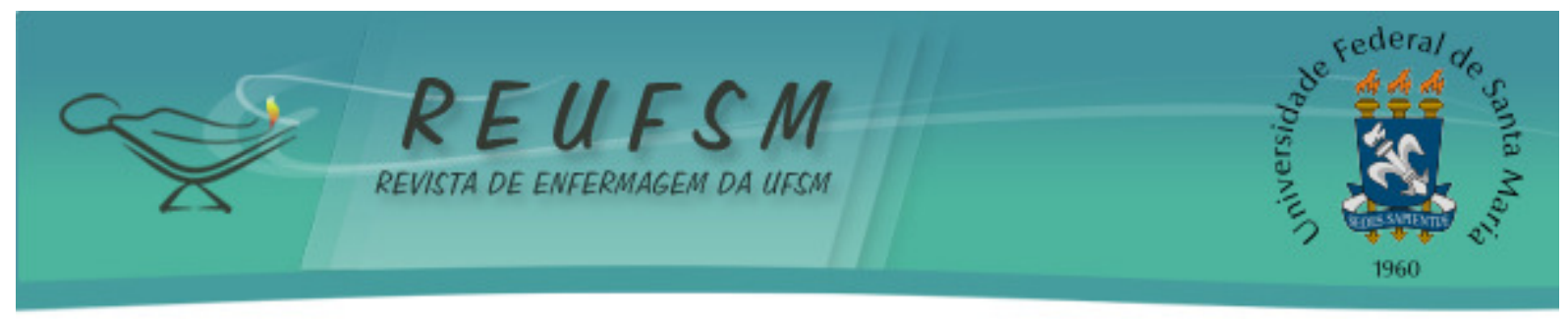

ARTIGO ORIGINAL

\title{
FRAGILIDADE EM IDOSAS RESIDENTES DE UMA INSTITUIÇÃO DE LONGA PERMANÊNCIA
}

\author{
FRAGILITY IN ELDERLY RESIDENTS OF LONG-TERM CARE INSTITUTION \\ FRAGILIDAD EN ANCIANAS RESIDENTES EN INSTITUCIONES DE LARGA \\ PERMANENCIA
}

Doi: $10.5902 / 2179769211328$

\author{
Graciela Maria Carneiro Maciel $^{1}$ \\ Hilderjane Carla da Silva ${ }^{2}$ \\ Maria Célia de Freitas ${ }^{3}$ \\ Rejane Maria Paiva de Menezes ${ }^{4}$
}

RESUMO: Objetivo: identificar o nível de fragilidade de idosas institucionalizadas e, a partir daí, indicar os diagnósticos, intervenções e resultados de enfermagem. Método: estudo transversal, realizado nos meses de abril e maio de 2012, em uma Instituição de Longa Permanência para Idosas de Fortaleza. Foi aplicada a Escala de Fragilidade Edmonton composta por nove domínios. Utilizou-se North American Nursing Diagnosis Association International, Nursing Intervention Classification e Nusing Outcomes Classification para elencar diagnósticos, intervenções e resultados de enfermagem. Resultados: 24 idosas, $16(66,6 \%)$ eram frágeis. Destas, de acordo com nível, $7(29,1 \%)$ tinham fragilidade leve, 2 (8,3\%) fragilidade moderada, $7(29.1 \%)$ fragilidade severa. Os domínios da escala adotados foram: independência funcional, uso de medicamentos, continência e desempenho funcional, sendo indicados seis diagnósticos; intervenções e resultados. Conclusões: uso da escala possibilitou identificação dos níveis de fragilidade, como, também, a sugestão de diagnósticos, intervenções e resultados esperados.

Descritores: Enfermagem geriátrica; Idoso; Idoso fragilizado; Processos de enfermagem.

ABSTRACT: Objective: to identify the level of fragility of institutionalized elderly women and, thereafter, indicate diagnoses, interventions and nursing outcomes. Method: crosssectional study, conducted in April and May 2012, in a long-term institution for elderly women in Fortaleza. The Edmonton Frail Scale was applied, considering nine domains. The International Taxonomy of North American Nursing Diagnosis Association, Nursing Intervention Classification and Nursing Outcomes Classification were utilized to list the diagnoses, interventions and nursing outcomes. Results: 16 out of 24 elderlies $(66.6 \%)$ were fragile. According to the level of frailty, 7 (29.1\%) were mildly frail, 2 (8.3\%) were moderately frail, 7 (29.1\%) were severely frail. The adopted domains of the scale were: functional independence, medicine usage, continence and functional performance, which led to the identification of six nursing diagnoses; interventions and results. Conclusions: the use of the scale allowed identification of levels of fragility, as well as the suggestion of diagnoses, interventions and expected outcomes.

Descriptors: Geriatric nursing; Aged; Frail elderly; Nursing process.

1. Enfermeira. Mestranda em Enfermagem. Universidade Federal do Rio Grande do Norte. Natal, Rio Grande do Norte, Brasil. gra_maciel@hotmaiil.com.

2. Enfermeira. Mestre em Enfermagem. Universidade Federal do Rio Grande do Norte. Natal, Rio Grande do Norte, Brasil. hilderjanecarla@hotmail.com.

3. Enfermeira. Docente. Doutora em Enfermagem. Universidade Estadual do Ceará. Fortaleza, Ceará, Brasil. celfrei@hotmail.com.

4. Enfermeira. Docente. Doutora em Enfermagem. Universidade Federal do Rio Grande do Norte. Natal, Rio Grande do Norte, Brasil. rejemene@gmail.com. 


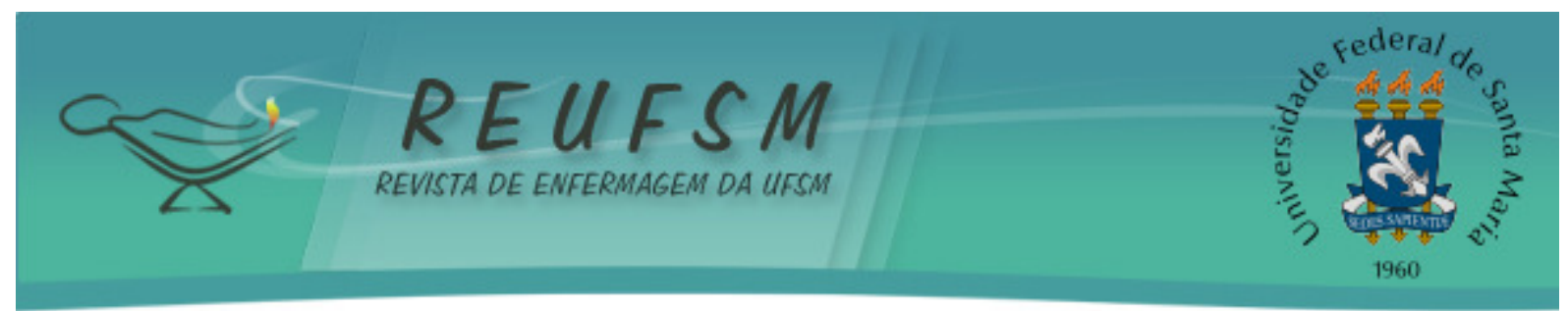

RESUMEN: Objetivo: identificar el nivel de fragilidad de ancianas institucionalizadas y, posteriormente, indicar diagnósticos, intervenciones y resultados de enfermería. Método: estudio transversal, realizado entre los meses de abril y mayo de 2012, en una institución de Larga Permanencia para Ancianas de Fortaleza (CE, Brasil). Se aplicó la Escala de Fragilidad Edmonton compuesta por nueve dominios. Se utilizó la North American Nursing Diagnosis Association International, Nursing Intervention Classification y Nusing Outcomes Classification para elencar diagnósticos, intervenciones y resultados de enfermería. Resultados: De 24 ancianas, 16 (66,6\%) eran frágiles. De éstas, según el nivel, 7 (29,1\%) tenían debilidad leve, 2 (8,3\%) fragilidad moderada, y 7 (29,1\%) fragilidad severa. Los dominios de la escala fueron: independencia funcional, uso de medicamentos, continencia y desempeño funcional, generando seis diagnósticos de enfermería, intervenciones y resultados. Conclusiones: el uso de la escala permitió identificar los niveles de fragilidad, y también sugerir diagnósticos, intervenciones y resultados esperados.

Descriptores: Enfermería geriátrica; Anciano; Anciano frágil; Procesos de enfermería.

\section{INTRODUÇÃO}

A fragilidade é considerada como uma síndrome multidimensional, que envolve fatores biológicos, físicos, cognitivos, sociais, econômicos e ambientais, ao longo da vida. ${ }^{1}$ Entre seus sinais e sintomas estão a diminuição da força muscular, perda de peso sem causa aparente no período de um ano, capacidade reduzida para atividade física, exaustão, alteração no tempo da marcha e do equilíbrio. ${ }^{2}$ Todos esses fatores contribuem para o risco de quedas, hospitalização, incapacidades, institucionalização, dependência e morte. ${ }^{3}$

Estima-se que de $10 \%$ a $25 \%$ das pessoas acima dos 65 anos e $46 \%$ acima dos 85 anos que vivem na comunidade sejam frágeis ${ }^{1}$, cuja maioria é do sexo feminino e tem idade maior que 80 anos. ${ }^{4} 0$ idoso que apresenta fragilidade necessita de uma atenção especial da equipe de saúde, principalmente quando se encontra em uma Instituição de Longa Permanência para Idosos (ILPI). Isto se torna importante porque uma ILPI é considerada como um ambiente que visa atender às necessidades dos idosos nos diferentes graus de dependência, diminuir e prevenir as morbidades, assim como promover um ambiente seguro para os seus residentes. ${ }^{5-6}$

A promoção integral à saúde da pessoa idosa exige que o enfermeiro da ILPI conheça o processo de envelhecimento e, em seu compromisso com o cuidado do ser humano, implemente estratégias que busquem manter ao máximo a autonomia e independência do idoso, na prestação de um cuidado individualizado ${ }^{5}$, especialmente àqueles que possuem fragilidades ou risco para desenvolvê-las.

Com a finalidade de melhorar a qualidade da atenção prestada aos residentes de ILPI, o uso de diagnósticos, intervenções e resultados de enfermagem possibilita ao enfermeiro aplicar os conhecimentos técnico-científicos, embasando a ação deste profissional e favorecendo o cuidado organizado.

Os sistemas de classificação de enfermagem possibilitam uma linguagem única e uma enfermagem clínica efetiva, como a classificação de diagnósticos de enfermagem North American Nursing Diagnosis Association International (NANDA-I), classificação de intervenções de enfermagem - Nursing Interventions Classification (NIC); classificação de resultados de enfermagem - Nursing Outcomes Classification (NOC). ${ }^{7}$

Estes sistemas de classificação fazem parte do Processo de Enfermagem. Assim, o cuidado prestado ao idoso embasado na história clínica, identificação dos diagnósticos, planejamento, intervenção e avaliação, na busca de ações planejadas e direcionadas às necessidades do indivíduo faz com que o cuidado seja de qualidade, abrangendo os aspectos físicos, mentais e sociais. ${ }^{8}$ 


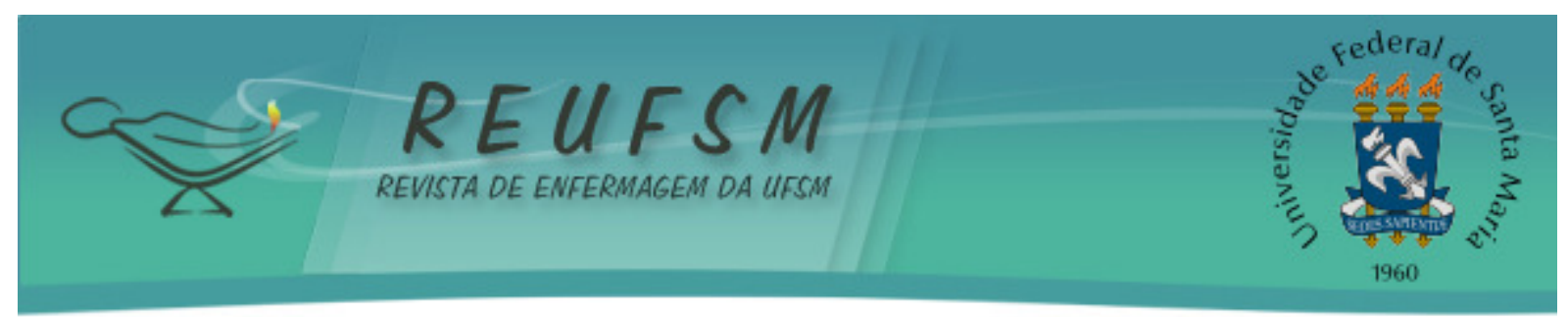

No entanto, a NANDA, NIC e NOC não são aplicadas em alguns contextos e grupos, a exemplo da assistência nas ILPIs, principalmente nos idosos que apresentam fragilidade. Assim os enfermeiros mostram-se despreparados ou não incentivados para prestação de uma assistência sistematizada e individualizada, através do processo de enfermagem, podendo comprometer o estado de saúde do idoso. ${ }^{9}$

Diante desta problemática, surgiu o seguinte questionamento: qual o nível de fragilidade das idosas institucionalizadas e quais são os diagnósticos, as intervenções e os resultados de enfermagem indicados diante do processo de fragilização no idoso?

Para responder a este questionamento, o presente estudo teve como objetivo identificar o nível de fragilidade de idosas institucionalizadas e, a partir daí, indicar os diagnósticos, intervenções e resultados de enfermagem.

\section{MÉTODO}

Trata-se de um estudo transversal descritivo, realizado em uma ILPI no município de Fortaleza/CE, cuja coleta de dados ocorreu nos meses de abril e maio de 2012. Esta ILPI foi fundada em 1980 com o intuito de receber freiras idosas que dependiam de cuidados ou em tratamento médico. A sua capacidade é para 30 idosas. Ela conta com o trabalho de oito técnicas de enfermagem, um fisioterapeuta e com outros serviços de saúde fora da instituição.

Utilizou-se como critérios de inclusão: ser idoso (idade igual ou superior a 60 anos); residir na ILPI selecionada. Critérios de exclusão: apresentar funções físicas, sensoriais e cognitivas que impossibilitassem responder ao instrumento de pesquisa.

A população do estudo foi composta por 29 idosas residentes no período do estudo, porém, nos meses da coleta de dados, duas idosas foram a óbito, duas se recusaram a participar da pesquisa e uma não correspondia à faixa etária considerada como idosa. A amostra final foi composta por 24 idosas.

Em primeiro lugar foi traçado o perfil pessoal, social (idade, frequência de visitas, escolaridade e tempo de institucionalização) e investigada a presença de comorbidades.

Em seguida foi aplicada a Escala de Fragilidade de Edmonton (EFE). A EFE é composta por 09 domínios: D1. Cognição (teste do desenho do relógio); D2. Estado geral de saúde (número de internações no último ano e autopercepção da saúde); D3. Independência funcional (número de atividades instrumentais de vida diária que precisa de ajuda); D4. Suporte social (conta com ajuda de alguém); D5. Uso de medicamentos (uso de cinco ou mais remédios e se esquece de tomar os remédios); D6. Nutrição (perda de peso); D7. Humor (sentimento de tristeza); D8. Continência (problema de perder o controle da urina) e D9. Desempenho funcional (teste "levante e ande" cronometrado). ${ }^{10}$

A soma de cada domínio dará o nível de fragilidade. Assim os escores para a análise da fragilidade são: 0-4, não apresenta fragilidade; 5-6, aparentemente vulnerável; 7-8, fragilidade leve; 9-10, fragilidade moderada; 11 ou mais, fragilidade severa. A pontuação máxima desta escala é 17 , que representa o nível mais elevado de fragilidade. ${ }^{10}$

Os dados obtidos foram registrados no Microsoft Excel e, em seguida, exportados para o Programa Statistical Package for the Social Sciences (SPSS) versão 16.0, na qual foi calculada a frequência absoluta e relativa, sendo apresentados através de tabelas.

Após a identificação dos níveis de fragilidade das idosas, foram utilizados os sistemas de classificação de enfermagem, através da sugestão dos principais diagnósticos, intervenções e resultados de enfermagem, conforme NANDA-I, a NIC e NOC de acordo com os quatro domínios da EFE (independência funcional, uso de medicamentos, continência e desempenho funcional). ${ }^{10-11}$ Esses domínios tiveram uma maior pontuação, influenciando o maior nível de fragilidade. 


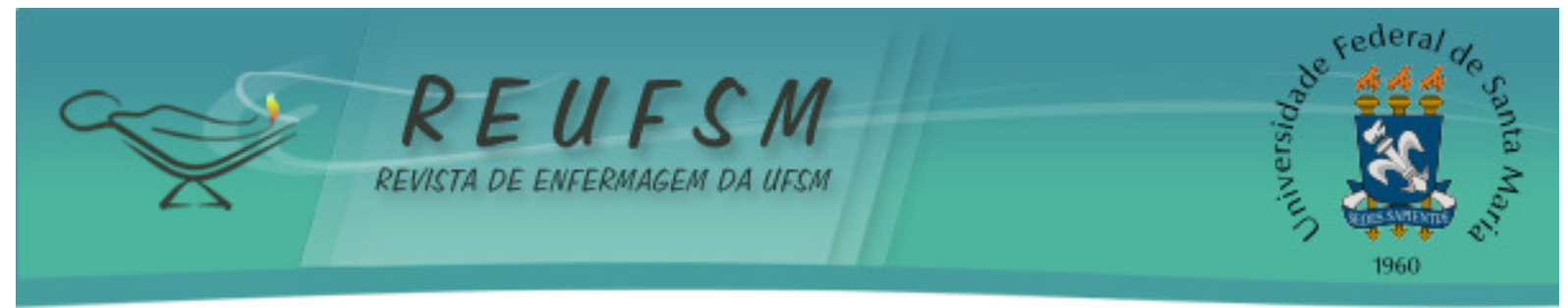

Em respeito à Resolução $196 / 96^{12}$, a pesquisa foi realizada após a aprovação do Comitê de Ética em Pesquisas da Universidade Estadual do Ceará, no dia 05 de janeiro de 2011, com protocolo $n^{\circ} 10029766-8$ e Folha de Rosto $n^{\circ} 331757$.

As idosas foram convidadas e esclarecidas sobre a pesquisa, seus objetivos, métodos, benefícios previstos, potenciais riscos, assegurando sua vontade de participar ou não do estudo antes da coleta de dados. Depois de lido e compreendido o Termo de Consentimento Livre e Esclarecido (TCLE) foi solicitada a assinatura da participante no TCLE, garantindo a manutenção do sigilo e da privacidade.

\section{RESULTADOS}

Foram prevalentes no estudo a faixa etária de 80 a 89 anos, 14 (58,3\%); escolaridade ensino superior, 14 (58,3\%); tempo de institucionalização até cinco anos, 10 $(41,6 \%)$ e recebimento de visitas, $17(70,8 \%)$ (Tabela 1$)$.

Tabela 1 - Perfil Pessoal e Social de idosas residentes em uma instituição de longa permanência para idosos (ILPI). Fortaleza, CE, 2012.

\begin{tabular}{lll}
\hline Variáveis & $\mathbf{N}$ & $\%$ \\
\hline Idade & 7 & $29,20 \%$ \\
$70-79$ & 14 & $58,30 \%$ \\
$80-89$ & 3 & $12,50 \%$ \\
$\geq 90$ & & $4,10 \%$ \\
Escolaridade & 1 & $37,50 \%$ \\
Ensino Fundamental & 9 & $58,30 \%$ \\
Ensino Médio & 14 & $41,60 \%$ \\
Ensino Superior & 10 & $33,30 \%$ \\
Tempo institucionalização & 8 & $25 \%$ \\
Até 5 anos & 6 & \\
Entre 5 e 10 anos & & $70,80 \%$ \\
Acima de 10 anos & 17 & $29,20 \%$ \\
Recebe visitas? & 7 & \\
Sim & & \\
Não & &
\end{tabular}

Conforme a Tabela 2 identificou-se que as comorbidades mais prevalentes entre as idosas institucionalizadas foram a hipertensão, 20 (83,3\%); as cardiopatias, 19 (79,1\%); e a osteoporose, 19 (79,1\%).

Tabela 2 - Presença de comorbidade em idosas residentes em uma instituição de longa permanência para idosos (ILPI). Fortaleza, CE, 2012.

\begin{tabular}{ccc}
\hline \multirow{2}{*}{ Comorbidades } & \multicolumn{2}{c}{ Sim } \\
\cline { 2 - 3 } & $\mathbf{n}$ & $\%$ \\
\hline Hipertensão & 20 & 83,3 \\
Cardiopatias & 19 & 79,1 \\
Osteoporose & 19 & 79,1 \\
Dislipidemia & 8 & 33,3 \\
Acidente vascular cerebral & 3 & 12,5 \\
Diabetes Mellitus & 2 & 8,3 \\
\hline
\end{tabular}




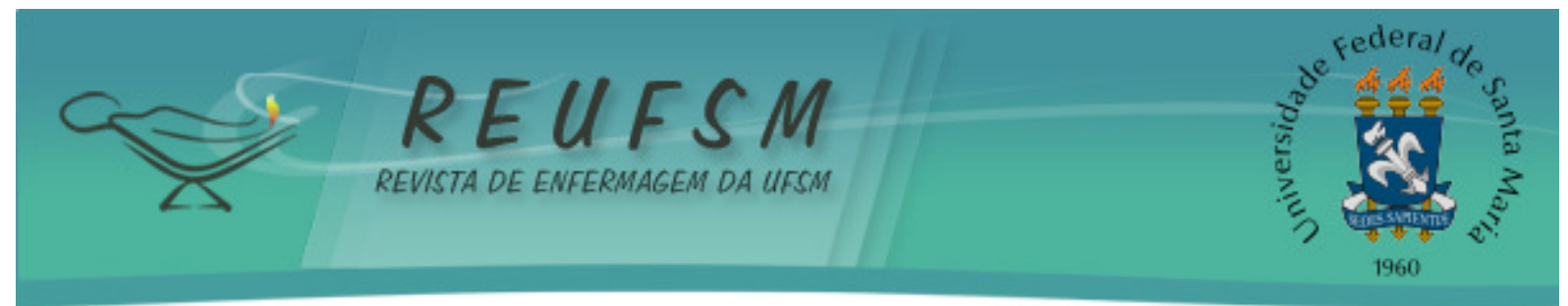

Após a aplicação da escala de fragilidade, identificou-se que tanto o nível fragilidade leve como fragilidade severa prevaleceram na pesquisa com 7 (29,1\%) idosas; seguidos de aparentemente vulnerável com 5 (20,8\%); não apresenta fragilidade com 3 $(12,5 \%)$ e $2(8,3 \%)$ com fragilidade moderada (Tabela 3$)$.

Tabela 3 - Nível de Fragilidade de idosas residentes em uma instituição de longa permanência para idosos (ILPI). Fortaleza-CE, 2012.

\begin{tabular}{lll}
\hline Variáveis & $\mathbf{n}$ & $\%$ \\
\hline Não apresenta fragilidade & 3 & 12,5 \\
Aparentemente vulnerável & 5 & 20,8 \\
Fragilidade leve & 7 & 29,2 \\
Fragilidade moderada & 2 & 8,3 \\
Fragilidade severa & 7 & 29,2 \\
\hline
\end{tabular}

Entre os nove domínios constituintes da EFE, os que apresentaram maior destaque no que condiz à fragilidade foram à Independência funcional, Uso de Medicação, Continência e Desempenho Funcional, visualizados na Tabela 4.

Tabela 4 - Classificação de fragilidade conforme os domínios da Escala de Fragilidade de Edmonton de idosas residentes em uma instituição de longa permanência para idosos (ILPI). Fortaleza, CE, 2012.

\begin{tabular}{|c|c|c|c|c|c|c|c|}
\hline \multirow[t]{3}{*}{ Variáveis } & \multicolumn{5}{|c|}{$\begin{array}{l}\text { Nível de fragilidade } \\
\end{array}$} & \multirow{2}{*}{\multicolumn{2}{|c|}{ Total }} \\
\hline & Ausente & Vulnerável & Leve & Moderada & Severa & & \\
\hline & $\mathbf{N}$ & $\mathrm{n}$ & $\mathbf{n}$ & $\mathrm{n}$ & $\mathrm{N}$ & $\mathbf{n}$ & $\%$ \\
\hline \multicolumn{8}{|l|}{ D1. Cognição } \\
\hline Aprovado & 2 & 4 & 3 & 0 & 1 & 10 & 41,6 \\
\hline $\begin{array}{l}\text { Reprovado com } \\
\text { erros mínimos }\end{array}$ & 1 & 1 & 2 & 1 & 1 & 6 & 25 \\
\hline $\begin{array}{l}\text { Reprovado com } \\
\text { erros significantes }\end{array}$ & 0 & 0 & 2 & 1 & 5 & 8 & 33,4 \\
\hline \multicolumn{8}{|l|}{$\begin{array}{l}\mathrm{D} .2 \mathrm{~N}^{\circ} \text { de } \\
\text { Internação }\end{array}$} \\
\hline 0 & 2 & 4 & 6 & 2 & 1 & 15 & 62.5 \\
\hline $1-2$ & 1 & 1 & 1 & 0 & 3 & 6 & 25 \\
\hline $\begin{array}{c}>2 \\
\begin{array}{c}\text { Autopercepção da } \\
\text { Saúde }\end{array}\end{array}$ & 0 & 0 & 0 & 0 & 3 & 3 & 12,5 \\
\hline $\begin{array}{c}\text { Exelente/ Muito } \\
\text { boa/ Boa }\end{array}$ & 3 & 4 & 3 & 1 & 1 & 12 & 50 \\
\hline Razoável & 0 & 1 & 4 & 0 & 1 & 6 & 25 \\
\hline Ruim & 0 & 0 & 0 & 1 & 5 & 6 & 25 \\
\hline \multicolumn{8}{|l|}{ D.3 Independência } \\
\hline funcional & & & & & & & \\
\hline $0-1$ & 3 & 1 & 1 & 0 & 0 & 5 & 20,8 \\
\hline $2-4$ & 0 & 3 & 2 & 1 & 1 & 7 & 29,1 \\
\hline
\end{tabular}




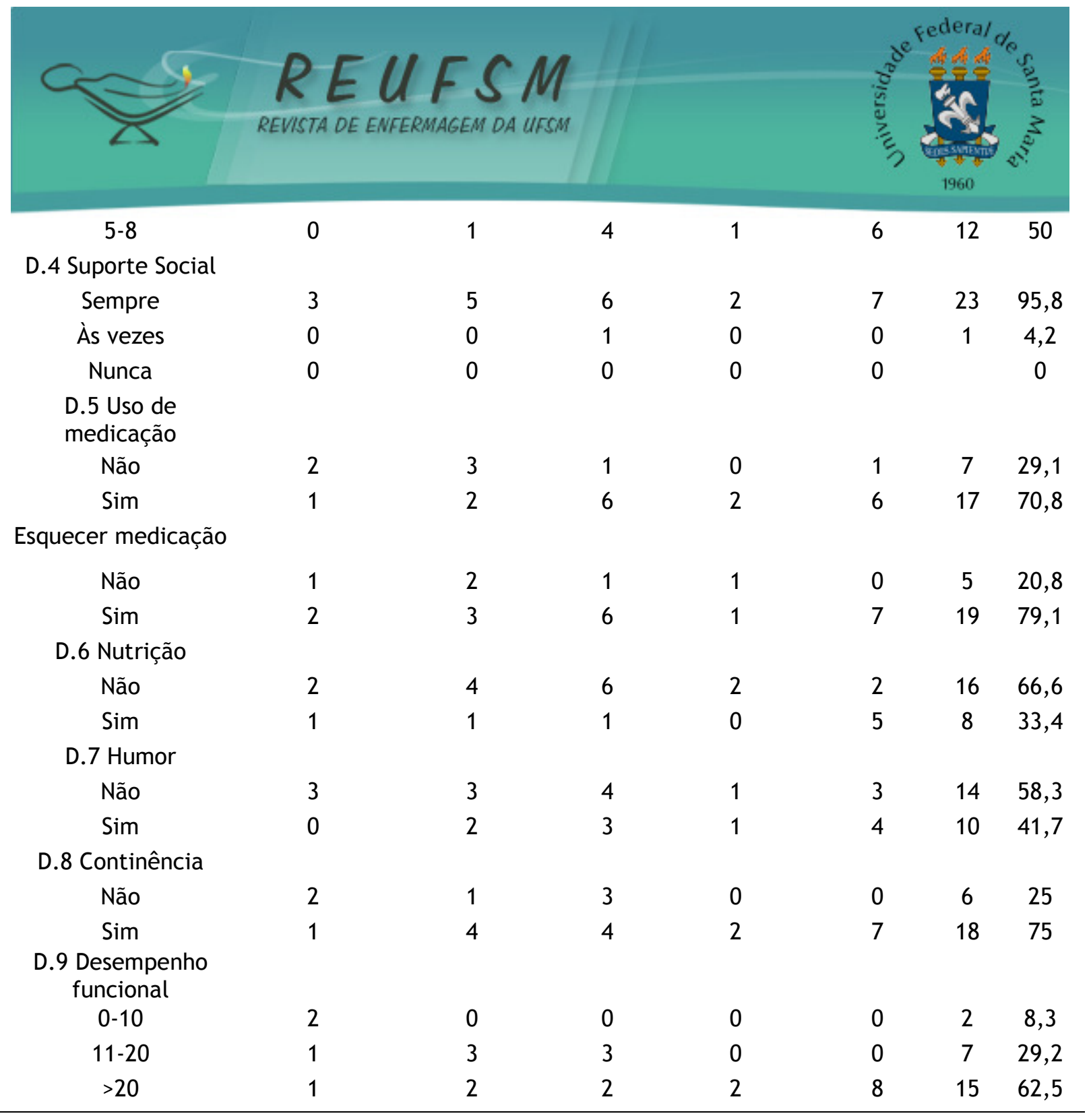

O domínio, correspondente a Independência Funcional, avaliou em quantas Atividades Instrumentais de Vida Diária (AIVD) a pessoa idosa necessita de ajuda, verificando que metade das idosas 12 (50\%) precisa de ajuda em 5 a 8 AIVD. Destas, 11 eram frágeis (Tabela 4).

Quanto ao uso da medicação, $17(70,8 \%)$ idosas consumiam acima de cinco remédios, destas 14 apresentavam fragilidade. Nesse mesmo domínio foi abordado o esquecimento dos horários de tomadas dos medicamentos e observou-se que quase 19 $(79,1 \%)$ das participantes não se lembravam de tomar os remédios na hora certa. Novamente, a maioria dessa categoria foi considerada com algum nível de fragilidade, como leve e severa (Tabela 4).

Em relação ao domínio Continência, 18 (75\%) das pesquisadas afirmaram ser incontinentes, das quais 13 eram frágeis (Tabela 4).

O ultimo domínio avaliado mostrou informações sobre o teste Levante e Ande (Timed up Go), na qual a maior parte, $15(62,5 \%)$, das idosas andou os três metros no tempo maior do que 20 segundos, havendo predomínio de fragilidade nesse grupo, conforme a Tabela 4.

A partir dos quatro domínios, de acordo com a EFE, foram indicados os principais diagnósticos, intervenções e resultados esperados de enfermagem, conforme as taxonomias NANDA, NIC e NOC, como pode ser visualizado no Quadro 1. 


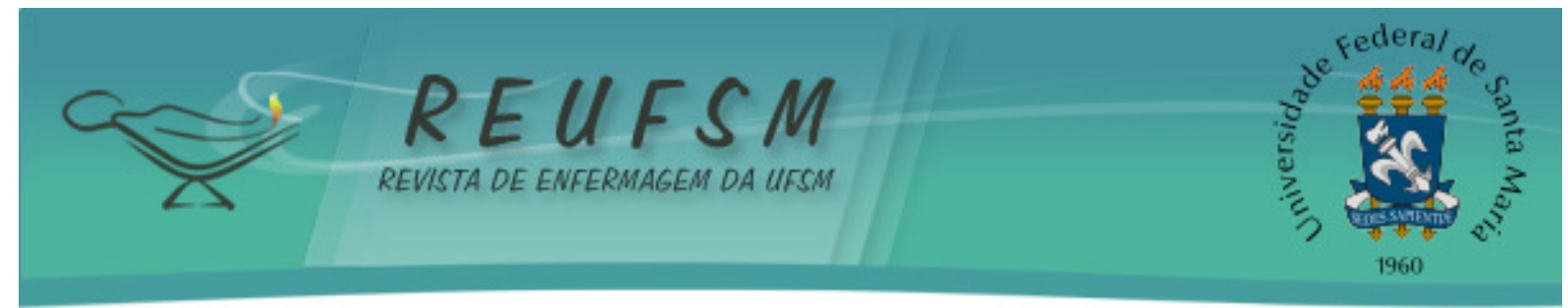

\begin{tabular}{|c|c|c|c|}
\hline $\begin{array}{l}\text { Domínios da } \\
\text { Escala de } \\
\text { Fragilidade }\end{array}$ & $\begin{array}{l}\text { Diagnósticos } \\
\text { de } \\
\text { Enfermagem }\end{array}$ & Intervenções & Resultados esperados \\
\hline $\begin{array}{c}\text { Independência } \\
\text { Funcional }\end{array}$ & $\begin{array}{c}\text { Intolerância à } \\
\text { atividade. }\end{array}$ & $\begin{array}{l}\text { Auxiliar no autocuidado } \\
\text { (alimentação, } \\
\text { banho/higiene, preparo do } \\
\text { lar), estimular exercícios } \\
\text { para controle e } \\
\text { fortalecimento muscular }\end{array}$ & $\begin{array}{l}\text { Autocuidado: capacidade } \\
\text { para desempenhar as } \\
\text { atividades da vida diária e } \\
\text { atividades instrumentais da } \\
\text { vida diária, de forma } \\
\text { independente, com ou sem } \\
\text { ajuda de acessórios }\end{array}$ \\
\hline $\begin{array}{c}\text { Uso de } \\
\text { Medicamentos }\end{array}$ & $\begin{array}{l}\text { Memória } \\
\text { prejudicada; } \\
\text { Déficit de } \\
\text { conhecimento } \\
\text { sobre o uso da } \\
\text { medicação. }\end{array}$ & $\begin{array}{l}\text { Ensinar sobre a importância } \\
\text { do uso dos medicamentos } \\
\text { prescritos e sobre o processo } \\
\text { de doença; facilitar a } \\
\text { aprendizagem. }\end{array}$ & $\begin{array}{l}\text { Extensão sobre } \quad \text { a } \\
\text { compreensão acerca do uso } \\
\text { seguro da medicação }\end{array}$ \\
\hline Continência & $\begin{array}{l}\text { Eliminação } \\
\text { urinária } \\
\text { alterada. }\end{array}$ & $\begin{array}{l}\text { Assistir o paciente no uso do } \\
\text { vaso sanitário; } \\
\text { Realizar o controle hídrico e } \\
\text { do uso de medicamentos; } \\
\text { Realizar sondagem vesical } \\
\text { (se necessário) }\end{array}$ & $\begin{array}{l}\text { Controle da eliminação de } \\
\text { urina }\end{array}$ \\
\hline $\begin{array}{l}\text { Desempenho } \\
\text { Funcional }\end{array}$ & $\begin{array}{l}\text { Risco para } \\
\text { queda; } \\
\text { Mobilidade } \\
\text { Física } \\
\text { Prejudicada. }\end{array}$ & $\begin{array}{l}\text { Aumentar a segurança do } \\
\text { ambiente; ensinar exercícios } \\
\text { de alongamento e equilíbrio, } \\
\text { prevenir quedas }\end{array}$ & $\begin{array}{l}\text { Comportamento de } \\
\text { segurança pessoal: esforços } \\
\text { do indivíduo ou do cuidador } \\
\text { para controlar } \\
\text { comportamentos capazes de } \\
\text { causar lesãor física; } \\
\text { Capacidade para manter o } \\
\text { equilibrio, locomoção, } \\
\text { mobilidade, movimento } \\
\text { coordenado e resistência }\end{array}$ \\
\hline
\end{tabular}

Quadro 1 - Propostas de diagnósticos, intervenções e resultados de enfermagem para o cuidado de idosas fragilizadas residente em uma instituição de longa permanência para idosos (ILPI). Fortaleza, CE, 2012.

\section{DISCUSSÃO}

A fragilidade, embora acometa os indivíduos com idade mais avançada, não deve ser entendida como sinal de velhice, pois pode ocorrer em qualquer idade, a depender de outros fatores de risco associados para o desenvolvimento da síndrome. ${ }^{10}$

Nesse estudo prevaleceram idosas com idade igual ou maior de 80 anos e tempo de institucionalização menor de cinco anos. Um estudo realizado em 2009, identificou que a idade igual ou superior a 75 anos e a institucionalização são fatores de risco para o desenvolvimento da fragilidade. ${ }^{13}$

A institucionalização acelera e/ou acentua a velocidade das perdas funcionais dos idosos, perda da autonomia e aumento da dependência, forçando o declínio das funções físicas e cognitivas, afastando-os da vida além dos muros da instituição. ${ }^{5}$

Em relação aos domínios da EFE, independência funcional avaliou a realização das AIVD. As AIVDs consistem as habilidades dos idosos em administrar o ambiente em que vivem, incluindo as seguintes ações: preparar refeições, fazer tarefas domésticas, lavar roupas, manusear dinheiro, usar o telefone, tomar medicações, fazer compras e utilizar os meios de transporte. ${ }^{14}$ 


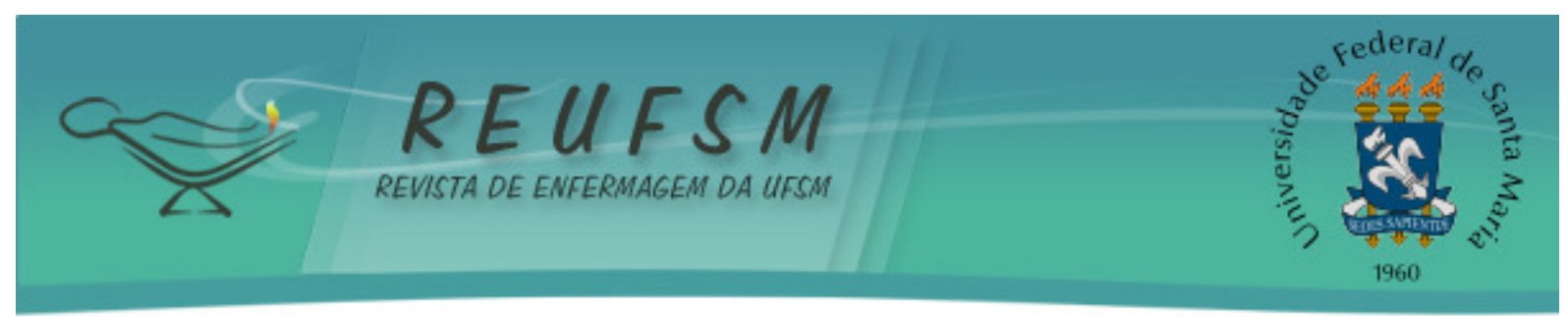

Alguns fatores podem interferir na independência dessas atividades como: as limitações visuais, auditivas, motoras e intelectuais, bem como o surgimento de doenças crônicas. ${ }^{14}$

No presente estudo, as idosas relataram a necessidade de ajuda em algumas AIVD. Os problemas sensoriais e dificuldade de locomoção estavam entre os maiores obstáculos para a realização das atividades, pois elas sentiam dificuldade em escutar a voz da outra pessoa no telefone, ver os nomes das medicações e mover-se, devido à presença de dores nas articulações, principalmente nos membros inferiores, o que prejudica a execução das tarefas.

Assim o diagnóstico sugerido, intolerância à atividade, e as intervenções de enfermagem; auxiliar no autocuidado, estimular exercícios para controle e fortalecimento muscular podem levar a resultados como: capacidade para desempenhar as atividades da vida diária e atividades instrumentais da vida diária, de forma independente, com ou sem ajuda de acessórios.

No domínio uso da medicação, o grupo das idosas que consumia mais de cinco medicamentos foi predominante sobre as que apresentavam fragilidade. Esses dados podem estar associados ao número de comorbidades, como Hipertensão, Diabetes Mellitus, Cardiopatias, Dislipidemias, Osteoporose identificados entre as institucionalizadas. Estudo realizado em 2008 relata que a fragilidade e o crescente número de medicações estão relacionados, principalmente, em decorrência do aumento das comorbidades, o que exige um maior número de medicamentos. ${ }^{13}$

Uma pesquisa sobre o perfil de consumo de medicamentos por idosos encontrou que $91 \%$ da amostra utilizavam, em média, 3,2 medicamentos, cuja classe terapêutica mais utilizada foi a de fármacos para o sistema cardiovascular. ${ }^{15}$

A exposição a múltiplos medicamentos e organização diante dos horários de administração dos fármacos, além da capacidade cognitiva afetada no paciente idoso, faz com que ocorra certa dificuldade para entendimento e/ou para a recordação correta dos seus regimes terapêuticos prescritos. ${ }^{15}$ Fato este evidenciado no estudo, pois a maioria das participantes da pesquisa não se lembrava de tomar os remédios no horário prescrito.

Durante a realização do estudo, observou-se que algumas idosas além de não saber o horário, também não se lembravam do nome das medicações, bem como sua finalidade. Vale salientar ainda a acomodação, devido à existência de uma cuidadora, que se responsabilizava por administrar as medicações nos horários corretos. Assim, esse grupo não era estimulado a aprender sobre o regime terapêutico e sua importância para a saúde.

Foram indicados dois diagnósticos: memória prejudicada e déficit de conhecimento sobre o uso da medicação. Assim, como intervenção sugere-se ensinar a importância do uso dos medicamentos prescritos e sobre o processo de doença, facilitando a aprendizagem e procurando obter resultados na compreensão acerca do uso seguro da medicação.

De acordo com o domínio continência, prevaleceram as idosas que possuíam instabilidade da bexiga, o que para alguns autores, tem causa multifatorial, como a idade avançada, problemas pélvicos, atrofia dos músculos e tecidos, queda funcional do sistema nervoso, circulatório e diminuição do volume vesical. ${ }^{16}$ Todos esses fatores afetam a função esfincteriana, reduz a elasticidade e contratilidade da bexiga, causando incontinência.

De acordo com o domínio citado anteriormente, o diagnóstico sugerido foi eliminação urinária alterada, na qual a intervenção indicada pode ser na assistência do paciente no uso do vaso sanitário; controle hídrico; do uso de medicamentos e na sondagem vesical, tendo como resultado o controle da eliminação de urina.

O último domínio, Desempenho Funcional, avaliou a mobilidade e a capacidade de deslocamento do indivíduo pelo ambiente, sendo um componente físico importante e um pré-requisito para a execução das atividades de vida diária (AVD), AIVD e a manutenção da independência.

As alterações na mobilidade dos idosos podem estar associadas às quedas, fraturas e institucionalização. 0 medo de cair tem consequências negativas no bem-estar físico e funcional, no grau da dependência, na capacidade de realizar normalmente as atividades, 


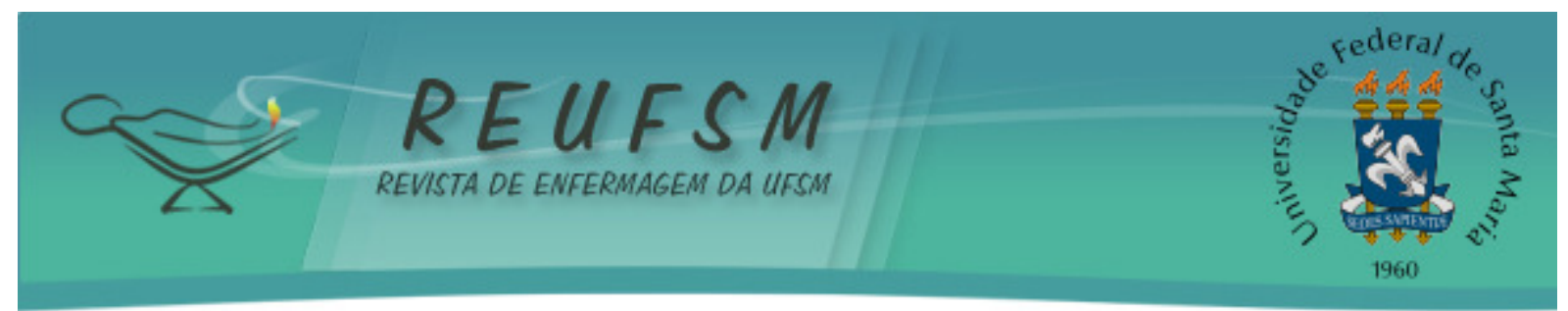

o que explica o grau de prevalência do estilo de vida sedentário nos idosos. ${ }^{17}$ Muitos destes se acomodam a ficar sentados, deitados e dependentes de andador/bengala.

Outro fator que prejudica a mobilidade do idoso é o uso de medicamentos que provocam efeitos colaterais, como tonturas e diminuição dos reflexos e perda de equilíbrio, cujas consequências podem ser as quedas e fraturas. ${ }^{18}$

Foram indicados assim, dois diagnósticos: risco para queda e mobilidade física prejudicada. Já as intervenções foram em relação à segurança do ambiente, exercícios de alongamento e equilíbrio, prevenção de quedas, tendo como resultados a segurança pessoal e capacidade para manter o equilíbrio, locomoção, mobilidade, movimento coordenado e resistência.

Diante disso, para a identificação das situações de risco que podem comprometer a saúde da pessoa idosa fragilizada, faz-se necessária a prestação de uma assistência integral, ao se considerar a pessoa idosa em sua multidimensionalidade, ou seja, em seus aspectos físicos, sociais e psicológicos. ${ }^{5,8}$

\section{CONCLUSÕES}

O uso da EFE possibilitou a identificação de idosas não frágeis e nos diferentes níveis de fragilidade. 0 cuidado prestado a essa população deve ter em vista a manutenção da autonomia, independência ou a diminuição de agravos, respeitando o nível da fragilidade, a idade e limites individuais.

Identificou-se uma necessidade de intervenções direcionadas, principalmente, às ações educativas de promoção da saúde e prevenção de agravos.

O tamanho da amostra constitui-se como uma das limitações para a realização do presente estudo, pois dificultou a generalização dos resultados. Vale salientar ainda que todos os participantes da pesquisa foram do sexo feminino, o que impediu a comparação com a fragilidade em homens.

Entende-se haver necessidade de uma maior investigação acerca da identificação da fragilidade e, portanto, sugere-se que novas pesquisas sejam realizadas com a aplicação desta escala em idosos de ambos os sexos, com idade inferior a 75 anos, que vivem na comunidade, a fim de identificar, prevenir ou minimizar a fragilidade nesta população.

Porém, ressalta-se que os resultados trazidos, embora com limitações, foram importantes como subsídios a prática de enfermagem, tendo em vista o cuidado com os idosos institucionalizados.

Alguns diagnósticos, intervenções e resultados foram indicados de acordo com quatro domínios da EFE, porém é importante destacar que esses sistemas de classificação fazem parte do processo de enfermagem. Este deve ser efetivado de forma completa, individualizada e não generalizada, devendo seguir todas as etapas.

\section{REFERÊNCIAS}

1. Teixeira INDO. Percepções de profissionais de saúde sobre duas definições de fragilidade no idoso. Ciênc Saúde Coletiva. 2008;13(4):1181-8.

2. Linck CL, Crossetti MGO. Fragilidade no idoso: o que vem sendo produzido pela enfermagem. Rev Gaúch Enferm [Internet]. 2011 [acesso em 2013 abr 12];32(2):385-93. Disponível em: http://www.scielo.br/scielo.php?pid=\$1983-14472011000200024\&script=sci_arttext.

3. Oliveira LPBA, Menezes RMP. Representações de fragilidade para idosos no contexto da estratégia saúde da família. Texto \& Contexto Enferm. 2011;20(2):301-9.

4. Santos EGS. Perfil de fragilidade em idosos comunitários de Belo Horizonte: um estudo transversal [dissertação]. Belo Horizonte: Universidade Federal de Minas Gerais, Escola de Educação Física, Fisioterapia e Terapia Ocupacional; 2008. 


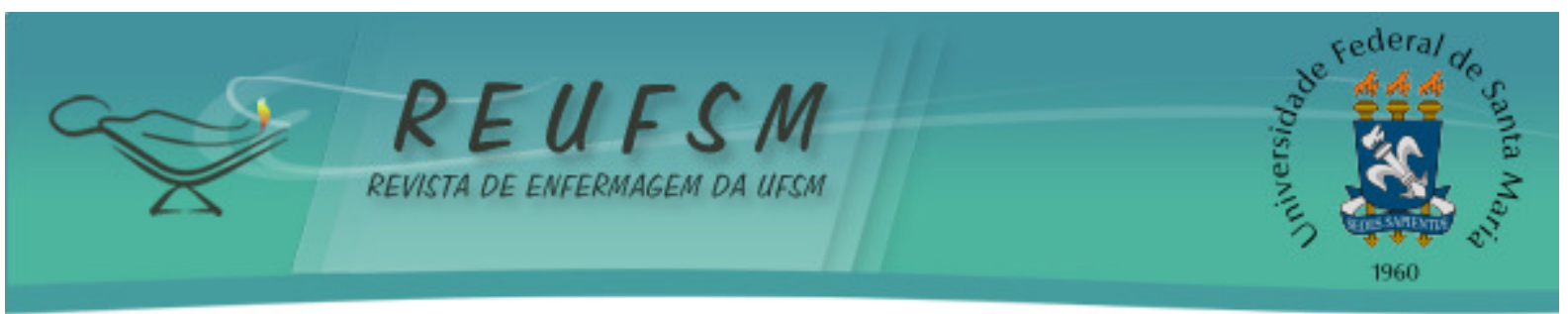

5. Santos SSC, Silva BT, Barlem ELD, Lopes RS. O papel do enfermeiro na instituição de longa permanência para idosos. Rev Enferm UFPE online [Internet]. 2008 [acesso em 2013 abr 10];2(3):291-9. Disponível em: http://repositorio.furg.br:8080/bitstream/handle/1/1537/PDF\%20n\%C2\%BA\%2018.PDF?sequence=1.

6. Creutzberg M, Gonçalves LHT, Sobottka EA. Instituição de longa permanência para idosos: a imagem que permanece. Texto \& Contexto Enferm. 2008;17(2):273-9.

7. Furuya RK, Nakamura FRY, Gastaldi AB, Rossi LA. Sistemas de classificação de enfermagem e sua aplicação na assistência: revisão integrativa de literatura. Rev Gaúch Enferm. 2011;32(1):167-75.

8. Cavalcante RB, Otoni A, Bernardes MFVG, Cunha SGS, Santos CS, Silva PC. Experiências de sistematização da assistência de enfermagem no Brasil: um estudo bibliográfico. Rev Enferm UFSM [Internet]. 2011 [acesso em 2014 mar 19];1(3):461-71. Disponível em: http://cascavel.ufsm.br/revistas/ojs-2.2.2/index.php/reufsm/article/view/2832/2396.

9. Oliveira DN, Gorreis TF, Creutzberg M, Santos BRL. Diagnósticos de enfermagem em idosos de instituição de longa permanência. Rev Ciência \& Saúde. 2008;1(2):57-63.

10. Fabrício-Wehbe SCC, Schiaveto FV, Vendrusculo TRP, Haas VJ, Dantas RAS, Rodrigues RAP. Adaptação cultural e validade de Edmonton Frail Scale - EFS em uma amostra de idosos brasileiros. Rev Latinoam Enferm. 2009;17(9):1043-9.

11. Johnson M, Bulecheck $G$, Butcher $H$, Dochterman JM, Maas $M$, Moorhead $S$, et al. Ligações entre NANDA, NOC e NIC: diagnósticos, resultados e intervenções de enfermagem. $2^{\mathrm{a}}$ ed. Porto Alegre: Artmed; 2009.

12. Ministério da Saúde (BR). Conselho Nacional de Saúde. Resolução CNS n 196, de 10 de outubro de 1996. Diretrizes e normas regulamentadoras de pesquisa envolvendo seres humanos. Brasília (DF): Ministério da Saúde; 1996.

13. Duarte YAO. Indicadores de fragilidade em pessoas idosas visando o estabelecimento de medidas preventivas. BIS, Bol Inst Saúde. 2009;(47):49-52.

14. Del Duca GF, Silva MC, Hallal PC. Incapacidade funcional para atividades básicas e instrumentais da vida diária em idosos. Rev Saúde Pública. 2009;43(5):796-05.

15. Werlang MC, Argimon IIL, Stein LM. Estratégias de memória utilizadas por idosos para lembrarem do uso dos seus medicamentos. Estud Interdiscip Envelhec. 2008;13(1):95-115.

16. Lazari ICF, Lojudice DC, Marota AG. Avaliação da qualidade de vida de idosas com incontinência urinária: idosas institucionalizadas em uma instituição de longa permanência. Rev Bras Geriatr Gerontol. 2009;12(1):103-12.

17. Lopes KT, Costa DF, Santos LF, Castro DP, Bastone AC. Prevalência do medo de cair em uma população de idosos da comunidade e sua correlação com mobilidade, equilíbrio dinâmico, risco e histórico de quedas. Rev Bras Fisioter. 2009;13(3):223-9.

18. Sachetti A, Vidmar MF, Silveira MM, Schneider RH, Wibelinger LM. Risco de quedas em idosos com osteoporose. Rev Bras Ciênc Saúde. 2010;(24):22-6.

Data de recebimento: $11 / 11 / 2013$

Data de aceite: 09/10/2014

Autor Correspondente: Graciela Maria Carneiro Maciel

Endereço Postal: Av. Abel Cabral 2400, AP-405, Residencial Spazio Nimbus, BL-05. Parnamirim,RN, Brasil. CEP: 59151-250. E-mail: gra_maciel@hotmail.com 